\title{
Optical fibre long period grating spectral actuators utilizing ferrofluids as outclading overlayers
}

\author{
Maria Konstantaki \\ mkonst@iesl.forth.gr
}

\section{Alessandro Candiani}

\section{Stavros Pissadakis}

\author{
Institute of Electronic Structure and Laser, Foundation for Research and Technology-Hellas, \\ P.O. Box 1385, 71110 Heraklion, Greece \\ Information Engineering Department, University of Parma viale G.P. Usberti 181/A, 43100 Parma, \\ Italy \\ Institute of Electronic Structure and Laser, Foundation for Research and Technology-Hellas, \\ P.O. Box 1385, 71110 Heraklion, Greece \\ Institute of Electronic Structure and Laser, Foundation for Research and Technology-Hellas, \\ P.O. Box 1385, 71110 Heraklion, Greece
}

Results are presented on the spectral tuning of optical fibre long period gratings utilizing water and oil based ferrofluids as outclading overlayers, under static magnetic field stimulus. Two approaches are adopted for modifying the ambient refractive index at the position of the long period grating. In the first approach, a water based ferrofluid is controllably translated along the length of the grating via a magnetic field. Changes as high as $7.5 \mathrm{~nm}$ and $6.5 \mathrm{~dB}$ are monitored in the wavelength and strength, respectively, of the attenuation bands of the grating. The repeatable performance of this device for repetitive forward and backward translation verifies that no ferrofluidic residue is left on the fibre, due to silanization cladding functionalisation. In the second approach, the refractive index of an oil based ferrofluidic overlayer is modified through the magneto-optical effect. For an applied static magnetic field in the order of 400 Gauss the strength of the attenuation band of the grating is modified by more than $10 \%$ while its spectral position remains unaffected. Accordingly for the implementation of the last approach, the magnetically induced refractive index changes of ferrofluids of different solution concentrations are studied by employing diffraction efficiency measurements. [D0I: 10.2971/jeos.2011.11007]

Keywords: optical fibres, long period gratings, ferrofluids, tunable filters, magnetic sensors

\section{INTRODUCTION}

Optical fibre long period gratings (LPGs) are key role components in a number of applications in the optical communication and sensing fields [1]. LPGs exhibit a periodic refractive index modulation, of the order of a few hundreds of microns that decouple the fundamental core mode to discrete forward-propagating cladding modes, which in turn predominantly interact with the cladding-environment interface $[2,3]$. Specifically, since the cladding modes interact with the surrounding material, changes in the optical or mechanical properties of this ambient material can straightforwardly be detected, rendering LPGs ideal for sensing applications.

There are several different examples where outcladding overlayers [4] and bulk media [5] have been applied onto LPGs for developing sensing [6] or simple switching devices [7]. Several methods have been reported in the literature for tuning LPGs through the properties of the ambient material, including thermo-optical [8] and magneto-optical effects [9]. In the work presented herein, the spectral response of a LPG is tuned by magnetically changing the optical/mechanical properties or the spatial distribution of a magnetosensitive outcladding fluid medium, namely, a ferrofluid. Ferrofluids are stable colloidal suspensions of sub-domain magnetic micro-/nano-particles dispersed in a suitable liquid carrier [10].
In the presence of a magnetic field, the magnetic moments of the particles orient along the field lines and magnetization of the fluid occurs. Such magnetisation results in viscosity, volume and density changes, as well as, in optical absorption and refractive index changes [11]. The nature of the dispersed magnetite nanoparticles renders ferrofluids optically opaque, allowing efficient propagation through them, in characteristic lengths of the order of few microns or less. In the past, ferrofluids have attracted significant attention due to their remarkable magneto-tuneability in applications such as microfluidic micropumps [12], in-line power modulators [13] or in adaptable lithographic techniques [14].

In the work presented here, two different methods were utilized to achieve magnetic tuning of the spectral features of the LPG. In the first approach the ferrofluidic outcladding is translated along the LPG length via a static magnetic field resulting in alterations of the spatial overlap of the outcladding ferrofluidic medium with the periodic perturbation. Hence, the transmission spectrum of the optical fibre is modified with respect to the ferrofluid position. A critical consideration for the above scheme, addressed for the first time here, is the repeatability of operation after repeat translations of the ferrofluidic outcladding. Repeatability could be jeop- 


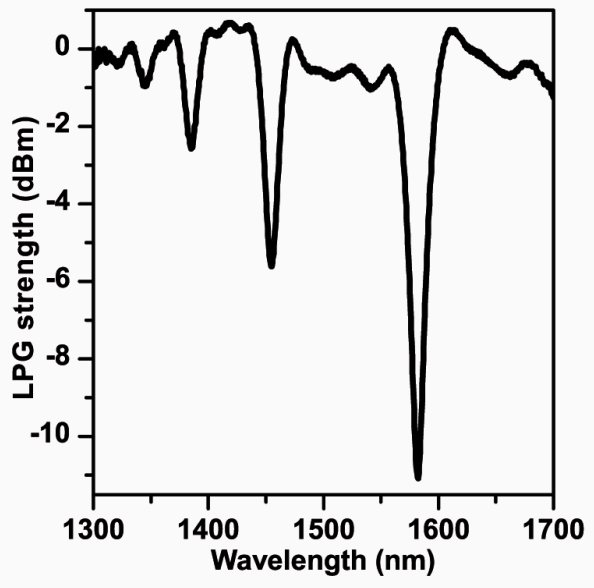

FIG. 1 Attenuation bands of the inscribed LPG in the $1300-1700 \mathrm{~nm}$ region.

ardized by the formation of ferrofluidic residue on the fibre therefore steps are taken to ensure that such residue formation does not occur. In the second scheme, the LPG was tuned through the modification of the refractive index of the magnetic fluid through the magneto-optical effect, while the ferrofluidic outcladding remains spatially static. In order to optimize the interaction between the ferrofluidic outcladding and the LPG, the ferrofluid was diluted with a suitable transparent solvent and results are presented for different solution concentrations. Within the frame of the last approach, the diluted ferrofluids used were characterized with respect to the refractive index changes induced under static magnetic field, by employing a simple diffraction efficiency liquid encapsulation setup.

\section{EXPERIMENTAL}

The LPGs used were inscribed utilizing a $\mathrm{KrF}$ excimer laser, emitting pulses with $34 \mathrm{~ns}$ duration at $248 \mathrm{~nm}$. The laser fluence incident on the fibre was $300 \mathrm{~mJ} / \mathrm{cm}^{2}$ per pulse, with a pulse repetition rate of $10 \mathrm{~Hz}$. Boron doped germanosilicate fibres (PS1250/1500 - Fibercore Ltd) were exposed through an amplitude mask made of titanium foil, with a $407 \mu \mathrm{m}$ period. For an exposure of approximately 100 seconds in the aforementioned conditions LPGs of up to $16 \mathrm{~dB}$ strength with $\approx 16 \mathrm{~mm}$ length are inscribed with attenuation bands observable in the $1300 \mathrm{~nm}-1700 \mathrm{~nm}$ region as shown in Figure 1.

Subsequently, the ferrofluids are applied on the LPGs as ambient materials according to the specific scientific approach adopted. To allow on-line monitoring of the attenuation bands of the LPG, light from a wide bandwidth superluminescent diode module is fed to the fibre and the output signal is monitored with an optical spectrum analyzer (OSA).

Two commercially available ferrofluids synthesized by Ferrotec USA, were used in the experiments performed here. The particles contained in both ferrofluids have an average size of $10 \mathrm{~nm}$ and are coated with a stabilizing dispersing agent which prevents particle agglomeration even when a
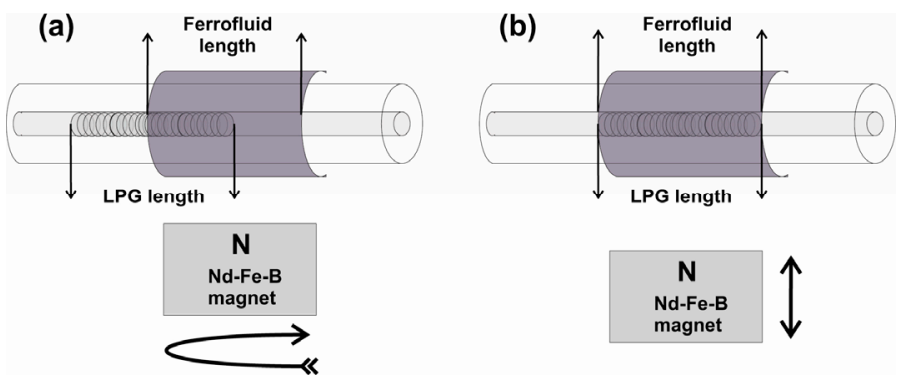

FIG. 2 Schematics of (a) a ferrofluidic long period grating actuator, and (b) a magneto -optical spectral tuning of long period grating utilizing spatially static ferrofluidic outcladding.

strong magnetic field gradient is applied to the ferrofluid. For the actuating medium approach a water based ferrofluid (EMG 605) was used, that has a 220 Gauss saturation magnetization, $5 \mathrm{mPa}$ viscosity $\left(27^{\circ} \mathrm{C}\right)$ and particle density $\left(25^{\circ} \mathrm{C}\right)$ equal to $1.18 \mathrm{~g} / \mathrm{cm}^{3}$. In the implementation of the magnetooptical approach a synthetic isoparaffinic solvent (oil based ferrofluid EMG 900) was used with 990 Gauss saturation magnetization and particle density $\left(25^{\circ} \mathrm{C}\right)$ equal to $1.74 \mathrm{~g} / \mathrm{cm}^{3}$ [15]. The refractive index of the water based ferrofluid (EMG 605 ) is estimated to be around $1.40 \pm 0.02$ while that of the oil based (EMG 900) slightly higher at $1.65 \pm 0.02$. To gain knowledge of the refractive index changes of the ferrofluids under a magnetic field stimulus, we characterized the materials using diffraction efficiency measurements. A thin layer of ferrofluid was applied on a fused silica relief phase mask optimized for $248 \mathrm{~nm}$ transmission. The ferrofluid was encapsulated into the phase mask grooves by pressing and fixing on its top a $100 \mu \mathrm{m}$ microscope slide. The above approach ensured that no liquid flow was taking place during magnetic field stimulation, while prevented evaporation of the ferrofluid. Applying different magnetic field strengths, the first order diffraction was then probed at the Bragg angle using a $632.8 \mathrm{~nm} \mathrm{He}-\mathrm{Ne}$ laser of random polarization. For the calculation of the equivalent refractive index change $\Delta \mathrm{n}$ induced on the ferrofluids, by magnetic field, standard diffraction efficiency theory was employed [16] and specifically the following equation:

$$
\Delta n=\arcsin \left(e^{\alpha d / \cos \Theta} \eta^{1 / 2}\right) \frac{\lambda \cos \Theta}{\pi d_{e f f}}
$$

were $\alpha$ is the absorption coefficient $\left(\mu \mathrm{m}^{-1}\right)$ of the sample at the probe wavelength $\lambda, d_{\text {eff }}$ is the effective thickness of the grating $\eta$ is the diffraction efficiency and $\Theta$ is the Bragg angle. The obtained results will be discussed in detail in latter sections.

In the first approach, the fibre section where the LPG is inscribed is immersed in a Ferrofluidic suspension. Specifically, the LPG is positioned and held straight inside a glass tube, containing the suspension, whose ends are sealed using silicon glue. In this suspension the ferrofluid is controllably moved along the length of the grating through an applied static magnetic field, using a bulk Nd-Fe-B magnet (see Figure $2 \mathrm{a}$ ) resulting in alterations of the spatial overlap of the 
outcladding ferrofluidic medium with the periodic perturbation.

A critical aspect in the implementation and evaluation of such a device that has not been addressed before [17] is the repeatability of operation; and specifically the deterioration of the LPG spectral response due to the adhesion of a residue layer of the ferro-active material on the cladding, following repeated passes. Indeed, the ferrofluids are colloids with significant content of surfactant, which always have high tendency to wet surfaces and to leave residue. Generally this can be reduced using specific glass material and mixing the ferrofluid with another liquid with low surface tension. During preliminary trials using LPGs with neat cladding surface, we verified that a thin ferrofluidic residue film was deposited onto the fibre interface after slow translation process. This residue was enough to distort the spectral response of the LPG and cancel repeatability, prerequisite for the applicability of such a device. In some cases, the residue had such a thickness as to be visible using an optical microscope.

In order to minimize the formation of residue on the fibre, different approaches were examined for the cases of oil and water based ferrofluids. After consideration of the properties of these materials, two experimental approaches were adopted: firstly, the modification of the physical properties of the surface of the fibre cladding to minimize the adhesion of ferrofluid on the glass surface, and secondly, the use of the ferrofluid in conjunction with another immiscible liquid that would assist in removing any residue on the fibre following the translation of the ferrofluid. Following various attempts it was concluded that optimum behaviour was obtained by employing a water based ferrofluid combined with a immiscible solution and a fibre with increased cladding surface hydrophobicity, reached through a procedure of silanization. For the silanization, the fibre was placed in a chamber of evaporating dichlorodimethylsilane $\left(\mathrm{C}_{2} \mathrm{H}_{6} \mathrm{C}_{12} \mathrm{Si}\right)$ for 24 hours at room temperature. Following this surface functionalization procedure, a hydrophobic layer of about $4 \mathrm{~nm}$ of silane $\left(\mathrm{SiH}_{4}\right)$ was formed on the LPG. The formation of this nano layer doesn't alter the transmission signal of the LPG.

\section{RESULTS AND DISCUSSION}

\subsection{FERROFLUIDIC LONG PERIOD GRATING ACTUATOR}

After the functionalization of the fibre, the LPG was positioned and held straight inside a glass tube of $70 \mathrm{~mm}$ length and $5 \mathrm{~mm}$ inner diameter whose ends were sealed using silicon glue. The tube was filled by $2 / 5$ with the water based ferrofluid EMG 605, while the rest $3 / 5$ were filled with a immiscible liquid which was a 50/50 mixture of Carbon Tetrachloride $\left(\mathrm{CCl}_{4}, \mathrm{n}=1.460\right)$ and Silicone oil (Dow Corning-705, $\mathrm{n}=1.579$ ). Therefore, out of the $70 \mathrm{~mm}$ of fibre inside the tube, approximately $28 \mathrm{~mm}$ were covered by the ferrofluid and $42 \mathrm{~mm}$ by the $\mathrm{CCl}_{4} /$ Silicone oil solution. The ferrofluid and the buffer $\mathrm{CCl}_{4} /$ Silicone oil solution were totally immiscible. The refractive index (n) of the $\mathrm{CCl}_{4} / \mathrm{Silicone}$ oil mixture was measured to be $1.52 \pm 0.01$ approximately. For controllable translation of the ferrofluid, the magnet was placed on a

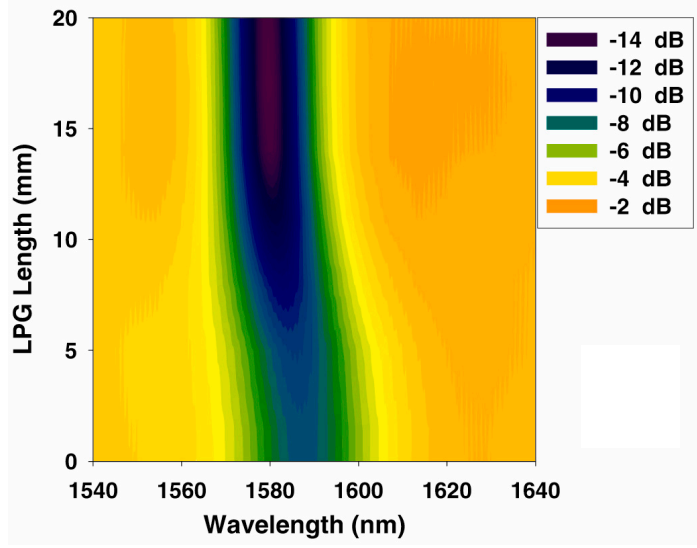

FIG. 3a Contour of the transmission spectra of the LPG attenuation band around 1587 $\mathrm{nm}$ for different overlapping with the water based ferrofluid.

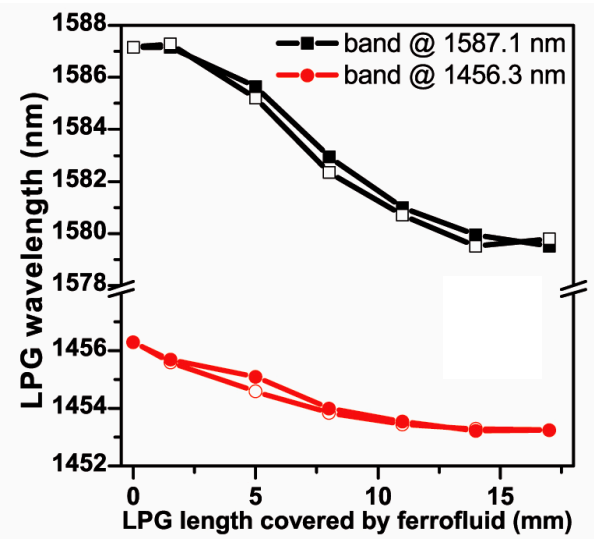

FIG $3 b$ Wavelength shift versus the LPG length covered by the ferrofluid for the two main attenuation bands of the LPG. The open square /circle curves correspond to data from repeated passes of the ferrofluid; negligible hysteresis is recorded.

motorized stage at a distance of $4 \mathrm{~mm}$ from the capillary tube. The magnetic field at the position of the LPG was 1000 Gauss, measured using a portable Gaussmeter (GM05, Hirst Magnetic Instruments Ltd). Translating the magnet parallel with respect to the capillary, the ferrofluid follows the movement of the magnetic field, covering a specific portion of the LPG length thus changing the spectral response of the grating.

The spectral response of the LPG with respect to the section of the LPG covered by the water based ferrofluid was monitored and recorded for all the attenuation bands in the spectral region $1300 \mathrm{~nm}-1700 \mathrm{~nm}$. Changes in both the strength and wavelength of the LPG notches were recorded. Specifically for the strongest attenuation band located at $1587.1 \mathrm{~nm}$, as shown on Figure 3a, a wavelength shift of $7.5 \mathrm{~nm}$ and a strength modulation greater than $6.5 \mathrm{~dB}$ in transmission is recorded between the two extreme cases of the LPG uncovered and fully covered by the ferrofluidic medium.

In the same conditions as shown in Figure $3 b$, the second attenuation band located at $1456.3 \mathrm{~nm}$ presented a $2.9 \mathrm{~nm}$ wavelength shift and $1.6 \mathrm{~dB}$ modulation in strength. Another dip was observed at $1383 \mathrm{~nm}$, but with negligible spectra 
changes. In order to confirm the repeatability of the performance, the magnet was translated for several cycles along the grating length, and negligible hysteresis was observed (see Figure $3 \mathrm{~b}$ open circle/ square plots), confirming the high efficiency of the cladding silanization process.

Simple experiments to evaluate the time response of the device were carried out by adapting the magnet to a motorize stage controlled by a DC power supply. By altering the voltage of the power supply different translation speeds of the stage and thus magnet and ferrofluid were achieved. The source used for these measurements was a tunable laser (Santec TSL-210) tuned at the minimum wavelength of the strongest attenuation band at $1568.4 \mathrm{~nm}$, (a new LPG is used for this set of measurements with slightly different attenuation band position). The light at the fibre output is fed to a photodiode connected to a digital oscilloscope. Results are reported in Figure 4 for speeds ranging from 7.6 to $13.5 \mathrm{~cm} / \mathrm{s}$. As can be seen, for speeds up to $8.1 \mathrm{~cm} / \mathrm{s}$ the actuation response of the device is constant in terms of extinction ratio. If the actuator is operated at higher speeds the maximum extinction ratio achieved starts to decrease, while during the first $10 \mathrm{~ms}$ of the actuation the signal is distorted probably due to uneven fluid transfer effects. The water based EMG 605 ferrofluid has a viscosity of $5 \mathrm{mPa} \cdot \mathrm{s} @ 27^{\circ} \mathrm{C}$, whilst that of the $\mathrm{CCl}_{4} /$ Silicone oil solution is more than double at $11 \mathrm{mPa} \cdot \mathrm{s} @ 22^{\circ} \mathrm{C}$. Previous flow studies presented by others [18] have shown that during the transfer of a low viscosity immiscible liquid into a sealed tube with a higher viscosity liquid, the lower viscosity liquid tends to encapsulate the higher one, leading to entrailment effects. This entrailment effect falls into a stable regime, when the volume ratio between the higher viscosity liquid versus the lower viscosity one is greater than a figure of $\sim 0.49$; parameter which is satisfied in the volume mixture ration applied herein. Therefore, in the present case, the low viscosity liquid -namely the water based ferrofluid- will detach from the long period grating cladding, while moving towards the outer glass capillary, ceasing its effect on the long period grating spectral tuning. Such an assumption can also be supported by the speed response data of Figure 4, where for the current arrangements and for speeds up to $9.5 \mathrm{~cm} / \mathrm{s}$ the device is operational, while the response is completely cancelled above $11.8 \mathrm{~cm} / \mathrm{s}$.

Although the time response illustrated in Figure 4 is slow in terms of telecom time frames it is still sufficient for a number of other sensing and actuating applications, including monitoring of magnetic valves, or flow speed and direction probing in microfluidic circuits. Further experiments are in progress for studying solutions that can interact better with the ferrofluid to improve the time response of the device.

\subsection{MAGNETO-OPTICAL TUNING OF A LONG PERIOD GRATING}

The second approach in the magnetic tuning of an LPG is based on the modification of the refractive index of a ferrofluid through the magneto-optical effect [8]-[10]. A grating fabricated with similar characteristics as of the previous experiment was positioned and held straight inside a capillary

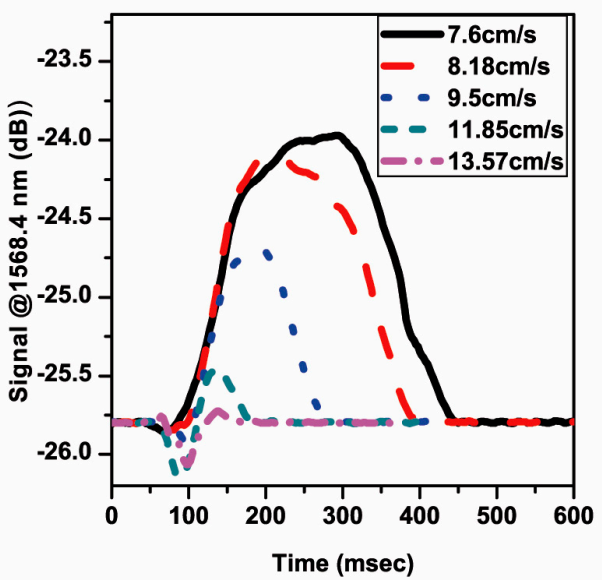

FIG 4 Transmitted signal measured at the wavelength of minimum transmission of the strong attenuation band for different translation speeds of the ferrofluid as described in the legend.

of $40 \mathrm{~mm}$ length and $1 \mathrm{~mm}$ inner diameter, filled with the oil based ferrofluid EMG 900. This ferrofluid was chosen based on the diffraction efficiency measurements of magnetically induced refractive index changes, since it was proven to posses the highest response among the ferrofluids studied; as well as, on its high refractive index value. Specifically, for the oil based ferrofluid reported in Figure 5a, the refractive index changes were calculated to be of the order of $-5 \times 10^{-2}$ when the magnetic field changes between 0 to 750 Gauss. Other oil based ferrofluids of lower particle concentration and thus density, gave lower refractive index changes of the order of $3.65 \times 10^{-2}$.

A bulk Nd-Fe-B magnet is placed initially at close proximity with the capillary (Figure $2 b$ ) while by displacing this magnet with respect to the fiber, thus increasing their distance, the magnetic field at the position of the ferrofluid is altered within the 0 to -1000 Gauss range.

As described above, the refractive index of the EMG 900 ferrofluid is $\approx 1.65$, much higher than the refractive index of the fiber. Previous reports with outclading overlays [5] indicate that significant spectral changes occur for outcladding media with refractive index close to that of the fibre silica cladding, namely the value of 1.446 . It was therefore decided that the ferrofluid should be diluted with an appropriate solvent in an attempt to lower its refractive index, and match that of the fibre cladding. EMG 900, contains $10 \mathrm{~nm}$ sub-domain magnetic particles in a oleic carrier, with a $16 \%$ iron oxide concentration. Volume dilution would decrease the iron oxide percentage in the solution and therefore decrease the refractive index changes induced under given magnetic field. However other optical properties of the ferrofluidic matrix such as refractive index and absorption loss shall be also modified. The dilution solvent provided by the manufacturers was an Isoparafinic Hydrocarbon with a refractive index of $1.44 \pm 0.01$. The results for solutions with a $10 \%, 20 \%$ and $30 \%$ concentration of the EMG 900 ferrofluid, respectively, are illustrated in Figure 5a.

Employing the diffraction efficiency measurement set-up described above the refractive indexes of the diluted ferroflu- 


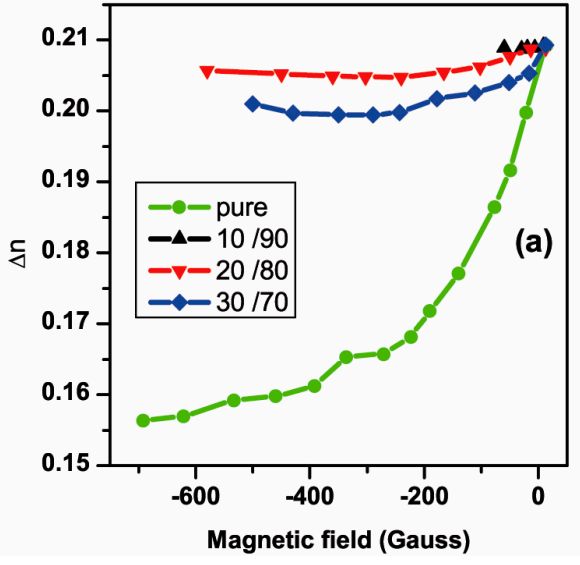

FIG. 5a Equivalent refractive-index change of the oil based ferrofluid for different solution concentration, under different magnetic fields determined by diffraction efficiency measurements. The legend describes the ferrofluid/solvent percentage with pure being the undiluted ferrofluid.

idic solutions were estimated to lay within a figure of 1.45 . Unfortunately the precision of the above technique in the absolute evaluation of the refractive index of liquids is rather limited, since it correlates diffraction efficiency measurements with refractive index changes and loss is a dominant factor. This specific inaccuracy becomes more pronounced in the case where almost opaque ferrofluidic solutions are measured for estimating their absolute refractive indexes. Therefore it is not possible to reliably discriminate between the refractive index of the three concentrations, due to the extreme absorption losses exhibited. This opaqueness also renders inadequate the employment of Abbe refractometer for the measurement. The transmission change of the LPG attenuation band (Figure $5 \mathrm{~b}$ ) located at $1540.7 \mathrm{~nm}$ for the $20 \%$ ferrofluid-solvent solution is $0.5 \mathrm{~dB}(\approx 11 \%)$ approximately for a magnetic field change of the order of 400 Gauss. The $20 \%$ concentration provides the optimum response since as it can be seen in the same figure the change is half for the $10 \%$ and $30 \%$ solutions. In all cases, changes only in the extinction ratio of the grating were observed, without any measurable spectral shifts of the attenuation bands. This behaviour is due to the absorption coefficient and refractive index changes given by the magneto-optical properties of the magnetic liquid, related with local ferrite nanoparticle density in the cladding vicinity. Employing ferrofluids with tailored refractive index would greatly enhance the monitored response of the device.

\section{CONCLUSIONS}

We have demonstrated spectral tuning of optical fibre long period gratings by utilizing ferrofluids as outcladding overlayers. Two different actuating approaches were presented; firstly, with the spatial, magnetically induced, displacement of the ferrofluidic medium, and secondly through magnetooptical refractive index change. Significant and consistent changes of the spectral behaviour were observed in both sys-

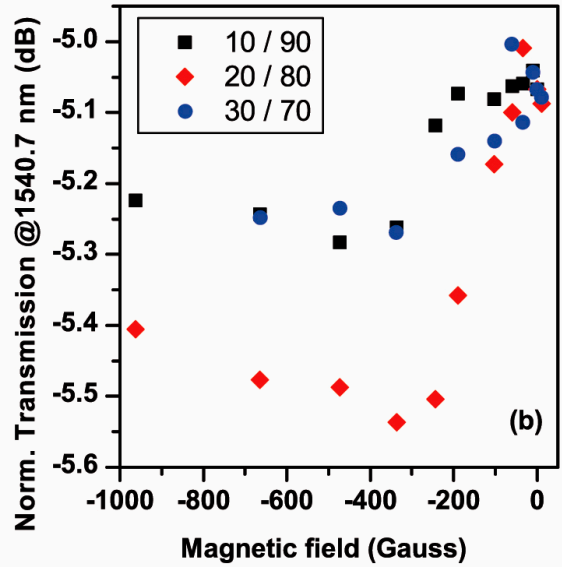

FIC. 5b Normalized transmission of the main attenuation band of the LPG for different ferrofluid solution concentrations versus magnetic field.

tems; specific and fundamental precautions were adopted, as surface fictionalization and fluid dilution, for optimizing the performances and for implementing high efficiency actuators and sensor. We are currently investigating the development of such ferrofluid transfer actuating units in microstructured optical fibres [19].

\section{ACKNOWLEDGEMENTS}

The authors would like to thank Dr. Franck Thetiot for advises related to the ferrofluid-oil suspension, and Dr. Emmanuel Stratakis (FORTH-IESL) for assistance with the fibre functionalization. SP would like to thank Dr Emmanuel Skarsoulis and Dr Yiannis Papaharilaou (FORTH-IACM) for useful discussions on the fluidic aspect of the actuating unit.

\section{References}

[1] S.W. James, R.P. Tatam, "Optical fiber long-period grating sensors: characteristics and application" Meas. Sci. Technol. 14, R49-R61 (2003).

[2] V. Bhatia, A.M. Vengsarkar, "Optical fiber long-period grating sensors" Opt. Lett. 21, 692-694 (1996).

[3] T. Venugopalan, T. Sun, K.T.V. Grattan, "Long period gratingbased humidity sensor for potential structural health monitoring", Sens. Actuator. A 148, 57-62 (2008).

[4] J. Lee, Q. Chen, Q. Zhang, K. Reichard, D. Ditto, J. Mazurowski, M. Hackert, S. Yin, "Enhancing the tuning range of a single resonant band long period grating while maintaining the resonant peak depth by using an optimized high index indium tin oxide overlay" Appl. Opt. 46, 6984-6989 (2007).

[5] H.J. Patrick, A.D. Kersey, F. Bucholtz, "Analysis of the response of long period fiber gratings to external index of refraction" J. Lightwave Technol. 16, 1606-1612 (1998).

[6] M. Konstantaki, S. Pissadakis, S. Pispas, N. Madamopoulos, N.A. Vainos, "Optical fiber long-period grating humidity sensor with poly(ethylene oxide)/cobalt chloride coating" Appl. Opt. 45, 4567-4571 (2006). 
[7] M. Konstantaki S. Pissadakis, "Optical fiber long period gratings with a photochromic overlayer" in Proceedings of ICOPHOTONICS, 55-58 (ICO, Delphi, 2009).

[8] K.R. Sohn, K.T. Kim, "Thermo-optically tunable band-rejection filters using mechanically formed long-period fiber gratings" Opt. Lett. 30, 2688-2690 (2005).

[9] T. Liu, X. Chen, Z. Di, J. Zhang, X. Li, J. Chen, "Tunable magnetooptical wavelength filter of long-period fiber grating with magnetic fluids" Appl. Phys. Lett. 91, 121116 (2007).

[10] R.E Rosenweig, Ferrohydrodynamics (Dover Publications Inc., Mineola, New York, 1997).

[11] H.E. Horng, C.-Y. Hong, S.Y. Yang, H.C. Yang, "Designing the refractive indices by using magnetic fluids" Appl. Phys. Lett. 82, 2434-2436 (2003).

[12] A. Hatch, A.E. Kamholz, G. Holman, P. Yager, K.F. Böhringer, "A ferrofluidic magnetic micropump" J. Micromech. Syst. 10,215221 (2001).

[13] H.E. Horng, J.J. Chieh, Y.H. Chao, S.Y. Yang, C.-Y. Hong, H.C. Yang, "Designing optical-fiber modulators by using magnetic fluids" Opt. Lett. 30, 543-545 (2005).
[14] B.B. Yellen, G. Fridman, C Friedman, "Ferrofluid lithography" Nanotechnology 15, S562-S565 (2004).

[15] www.ferrotec.com

[16] H.M. Smith (ed.), Holographic Recording Materials (SpringerVerlag, Berlin, 1977).

[17] W. Liao, X. Chen, Y. Chen, S. Pu, Y. Xia, Q. Li, "Tunable optical fiber filters with magnetic fluids" Appl. Phys. Lett. 87,151122 (2005).

[18] D.D. Joseph, M. Renardy, Y. Renardy, "Instability of the flow of two immiscible liquids with different viscosities in a pipe" J. Fluid Mech. 141, 309-317 (1984).

[19] A. Candiani, M. Konstantaki, W. Margulis, and S. Pissadakis, "A spectrally tunable microstructured optical fibre Bragg grating utilizing an infiltrated ferrofluid" Opt. Express 18, 24654-24660 (2010). 